Cinémas

Revue d'études cinématographiques

Journal of Film Studies

\title{
En deçà et au-delà du cinéma moderne. Visconti, mélancolie et néo-baroque
}

\section{Denilson Lopes}

Volume 8, numéro 1-2, automne 1997

Cinéma et mélancolie

URI : https://id.erudit.org/iderudit/024745ar

DOI : https://doi.org/10.7202/024745ar

Aller au sommaire du numéro

Éditeur(s)

Cinémas

ISSN

1181-6945 (imprimé)

1705-6500 (numérique)

Découvrir la revue

Citer cet article

Lopes, D. (1997). En deçà et au-delà du cinéma moderne. Visconti, mélancolie et néo-baroque. Cinémas, 8(1-2), 113-124. https://doi.org/10.7202/024745ar

\section{Résumé de l'article}

Le désir de fiction et la fascination pour le spectacle furent considérés, durant la période moderne, comme régressifs ou simplement académiques. Ce désir et cette fascination retrouvent aujourd'hui une valeur sous l'impulsion d'une nouvelle narrativité. Dans ce contexte, l'analyse du néo-baroque dans l'oeuvre tardive de Visconti permet d'y comprendre la construction de l'espace et du temps, la constitution de la subjectivité et la sensibilité mélancolique. 


\title{
En deçà et au-delà du cinéma moderne. Visconti, mélancolie et néo-baroque
}

\section{Denilson Lopes}

\begin{abstract}
RÉSUMÉ
Le désir de fiction et la fascination pour le spectacle furent considérés, durant la période moderne, comme régressifs ou simplement académiques. Ce désir et cette fascination retrouvent aujourd'hui une valeur sous l'impulsion d'une nouvelle narrativité. Dans ce contexte, l'analyse du néo-baroque dans l'cuvre tardive de Visconti permet d'y comprendre la construction de l'espace et du temps, la constitution de la subjectivité et la sensibilité mélancolique.
\end{abstract}

\section{ABSTRACT}

In the modern period, the desire for fiction and the fascination with spectacle were considered either regressive or purely academic. Today this desire and this fascination have regained their value under the impetus of a new narrativity. In this context, the analysis of the neo-baroque in Visconti's late work helps us to understand his construction of space and time, his constitution of subjectivity, and his melancholic sensibility.

Au début ce n'étaient que plusieurs images. Et parmi celles-ci s'en est détachée une, celle du Guépard de Luchino Visconti (1963) : à la fin de la fameuse séquence du bal, le prince Salina se regarde dans la glace et il pleure, sans mot dire. Ce n'est pas 
un jeune visage que le miroir lui renvoie, mais celui d'un homme qui se découvre vieux et fragile face au temps. Cette image résume bien ce film et elle figure tout aussi bien le fil conducteur de ce texte, la mélancolie.

La mélancolie est plus qu'un sentiment individuel tel qu'on l'entend généralement. Elle ne doit pas être envisagée uniquement dans sa connotation négative, comme une pathologie voisine de la dépression (Freud) ou de l'anomie (Durkheim). Je ne la vois pas non plus définie comme une perspective philosophique (nihilisme) ni comme un discours qui aurait parcouru différents savoirs. Elle n'est pas aussi statique qu'une mentalité ou qu'une vision du monde (histoire), elle ne se restreint pas à l'apathie comme idéologie de classes ou de groupes sociaux en décadence ou sans milieu social.

Au-delà de l'interprétation par Julia Kristeva de la mélancolie comme étant une potentialité de l'homme occidental dans la rupture/ hésitation entre nature et culture (p. 112-113), je reconceptualise ce phénomène de la mélancolie, en empruntant la voie de Walter Benjamin, pour lequel elle est une sensibilité sociohistorique bâtie à partir de l'expérience de l'écoulement du temps, de sa finitude, et ce dans la douloureuse difficulté d'oublier en un monde où excelle la vitesse (Matos, p. 21). Sensibilité antiutopique, la mélancolie se rattache à la mort comme catégorie existentielle centrale et à la catastrophe comme catégorie historique privilégiée. Le passé ne cesse de se perdre, le présent est plein de ruines et le futur est évidé de tout sens utopique. Ce souci du temps fait de la mélancolie un savoir qui se penche sur la matérialité des choses créées, un micro-regard qui met en relief le détail et le quotidien. Cette sensibilité saturnienne differe d'une sensibilité dionysiaque, tragique, qui aurait comme noyau le mythe ou le désir, accentuée par un temps circulaire ou par une extase libératrice de l'histoire. Elle differe aussi d'une sensibilité apollinienne, centrée sur la raison comprise comme rationalité scientifique et soutenue par un temps linéaire ou dialectique, en progression continue.

La vision benjaminienne se distingue fortement de celle de Freud. Pour Benjamin, la mélancolie est à la fois un savoir et une sagesse qui vient de l'abîme, de l'immersion dans le monde des 
choses créées (Rouanet, p. 17). Au contraire, Freud prend en compte la mélancolie comme forme pathologique du deuil, dans laquelle le sujet résout le conflit non par le désinvestissement graduel de l'objet perdu, mais par son incorporation psychique, sous la forme d'une régression narcissique (Rouanet, p. 37 et 40). Il y aurait, différemment du deuil, peu de netteté quant à ce qui a été perdu, alimentant ainsi une " $[\ldots]$ intention de deuil qui précède et anticipe la perte de l'objet" (Agamben, p. 48). "Dans le deuil, c'est le monde qui devient pauvre et vide; dans la mélancolie, c'est le moi lui-rnême" (Freud, p. 277-278). De cette perspective, Freud considère la mélancolie en tant que maladie (p. 279). En essayant d'établir une distinction bien tranchée entre deuil et mélancolie, il institue le deuil comme norme et non comme exception. "Ce qui semble étrange est la possibilité de pouvoir déclarer comme perdu l'objet de l'amour, cela même auquel on se trouvait identifié. Le deuil est une énigme" (Matos, p. 120).

Quoique l'histoire de la mélancolie soit antérieure à la civilisation occidentale et remonte au moins à la Grèce classique, ce n'est qu'à la modernité que la mélancolie émerge comme sensibilité, au fur et à mesure que l'individu devient une valeur centrale. La mélancolie se cristallise pour la première fois dans l'imaginaire du XVII siècle avec le baroque.

Le néo-baroque est une reprise au $\mathrm{XX}^{\mathrm{e}}$ siècle du baroque historique du XVII, et plus qu'une tendance artistique, il se constitue comme imaginaire. Ses images, narrativement et dynamiquement, mettent en scène la sensibilité d'un réseau et d'une constellation d'objets hétéroclites issus d'échanges transculturels. Cet imaginaire, en tant que moyen de connaissance, s'articule autour d'une conception du monde comme image, il reprend souffle face au développement des sociétés de masses au XXe siècle, et devient un instrument efficace pour explorer les rapports entre différentes expressions artistiques.

Le dialogue avec le baroque constitue un premier pas pour écrire l'histoire de la mélancolie au contemporain et constituer un passé qui résiste au rachat d'une tradition néo-illuministe qui détermine le XVIII ${ }^{e}$ siècle comme temps fondateur. Ce dialogue se distingue aussi d'une tradition antimétaphysique qui remonte à la Grèce préclassique, en même temps qu'il fuit la dualité

En deçà et au-delà du cinéma moderne. Visconti, mélancolie et néo-baroque 
modernitélpostmodernité dans l'analyse du contemporain en la déstabilisant.

La prise en considération du néo-baroque comme imaginaire essaie à la fois d'éviter une généralisation percevant le baroque comme une constante dans l'histoire humaine ainsi que les limitations temporelles étroites, ici le XVII ${ }^{\mathrm{e}}$ siècle, et les associations à des identités essentialistes concernant l'Amérique latine. Le baroque, par sa persistance sur le continent latino-américain, a favorisé l'émergence d'un projet alternatif et critique par rapport à la modernité européenne et nord-américaine. Le néo-baroque dévoile une double crise, celle du patriarcat et de l'individualisme, comme si en un même moment il y avait eu un début et une fin de la modernité dans le passage sans détour des sociétés traditionelles à la postmodernité, sans que nous ayons effectivement été modernes.

Le néo-baroque réaffirme une histoire de la mélancolie qui, sous la figure de l'allégorie, détruit et re-signifie des objets dans le temps, les renvoie à leur matérialité de choses en proie à l'anarchie et à leur irrévocable fugacité. L'allégorie concerne aussi bien le déploiement des images qui composent les personnages et le récit que cette nouvelle dimension de l'histoire faite d'images en ruine, une histoire imaginaire. La représentation la plus adéquate de l'allégorie dessine une spirale décroissante autour d'un axe qui n'est atteint qu'au moment où il n'y a plus d'histoire. Cet axe, d'après Walter Benjamin (p. 188), c'est la mort, son principe structurant ainsi que contenant.

Afin d'identifier la spécificité de la lecture du baroque en jeu dans les films de Visconti étudiés, j'ai d'abord établi une distinction entre deux néo-baroques. D'une part, un néo-baroque majeur, hyperbolique, plus proche de la tradition joycéenne d'une haute modernité, met en présence un humour de style parodique, novateur au niveau du langage, caractérisé par une économie de la dépense et une exaltation de l'érotisme, et ce avec une préférence accentuée pour les images oniriques et les relations intertextuelles puisées de sources orales et des médias de communication de masse, comme chez les écrivains Carpentier, Lezama Lima, Severo Sarduy et les cinéastes Glauber Rocha et Peter Greenaway. D'autre part, un néo-baroque 
mineur, corrosif, et non transgresseur, s'exprime dans le comportement hiératique des personnages, par la rigueur et la sobriété des mots et des images, au sein duquel le modèle réaliste est perverti par une allégorie en gestation. C'est cette deuxième forme qui attire ici mon attention, telle qu'elle se présente dans quelques films de Visconti, dont Le Guépard (1963), Ludwig (1973) et Violence et passion (1974).

Dans l'imaginaire néo-baroque de ces films de Visconti, la mélancolie s'incarne à travers des personnages allégoriques de princes et d'aristocrates sans pouvoir. Et ces personnages allégoriques appartiennent aussi bien aux textes artistiques qu'aux textes culturels. Ces personnages ne sont pas des types idéaux, des prototypes de groupes sociaux aux visées didactiques, mais bien des figures esthétiques, des blocs de sensations et d'images, des fictions théoriques incomplètes, ambiguës, qui changent dans le temps, se déploient en images autour d'un noyau vide, la mort, dans une situation de tension, d'intermédiaire, entre le monde traditionnel, rural et les sociétés urbaines, modernes, ou entre la modernité et la postmodernité.

Les protagonistes sont «[...] littéralement fécondés par l'histoire" (Sanzio et Thirard, p. 46), spectateurs privilégiés de la décadence de leurs moyens, transformés en ensembles historicosociaux comme dans un opéra (Daney et Oudart, p. 2). Ils ont une conscience poussée de l'histoire, mais avec cette liberté de pouvoir s'en éloigner et se tourner vers leur vie privée, comme le prince Fabrizio du Guépard. Sa quête décevante, échec face au temps, peut être résumée par le mot de T. S. Elliot: «Être conscient, c'est être hors du temps. "Quoique cette liberté puisse signifier la mort, elle n'invalide pas pour autant la maxime de Ludwig: "Je veux être libre de chercher le bonheur dans l'impossible." Et cela se vit plus dans l'appel à l'esthétisation de la vie que dans la force de la liberté de la mort dans la vie, contre toute norme, prudence, sécurité et médiocrité. Ou encore, le désir d'esthétique du professeur dans Violence et passion, quoique sous un mode mineur par rapport à celui de Ludwig, lui confere la mort pour " [...] avoir désiré doter de réalité les tableaux qu'il collectionne" (Ishaghpour, p. 21). Nous le voyons bien, la mélancolie n’appelle pas la vérité tel le ferait un texte

En deçà et au-delà du cinéma moderne. Visconti, mélancolie et néo-baroque 


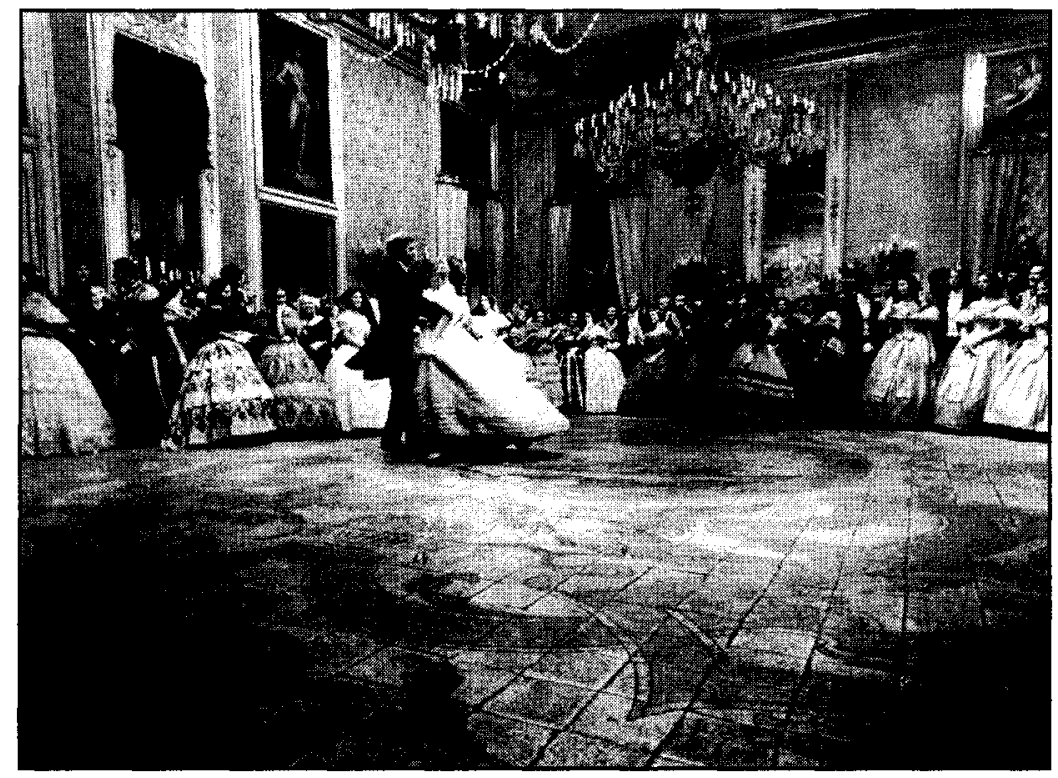

\section{Le Guépard de Luchino Visconti (1962)}

Collection Cinémathèque québécoise

social dictant une vérité pour tous, mais elle engage un ensemble d'affects et simule une stratégie pour en modérer l'effet réalité (McGraw, p. 82).

Le cinéma de Visconti s'érige en un autel pour icônes. Ses personnages sont à l'image même des acteurs, icônes forgés moyennant un dépassement du prosaïque par l'historique (Rocha, $\mathrm{p}$. 175), depuis l'image du prince Fabrizio de Salina lié à une éthique aristocratique inutile dans le monde bourgeois, à celle de Ludwig, profondément partagé entre une éthique esthétique et l'idée carholique du péché, et jusqu'à celle du professeur, attaché à une éthique humaniste, influencée par la gauche de l'aprèsguerre. Tous ces personnages ont la sensation d'être en retard, hors de leur temps. Il est trop tard pour qu'ils vivent dans le monde qui surgit et trop tôt pour qu'ils y soient compris.

La lecture baroque de Visconti veut mettre en relief le second moment de son ouvre cinématographique, ses derniers films, son style tardif, anachronique, alors qu'on l'accusait, en s'éloignant nettement du néo-réalisme, d'académisme pédant et con- 
servateur. Cette lecture vise aussi à construire un regard plus convaincant que celui du réalisme critique, en constituant comme modèle la fusion opérée entre le grand récit du $\mathrm{XIX}^{\mathrm{e}}$ siècle et le mélodrame populaire opératique. Elle se présente comme une tentative contraire à la modernité cinématographique des années soixante dans son projet de relier l'illusion, le spectacle et l'histoire, le cinéma et son public. Ce dilemme, si cher aux cinéastes contemporains pris par leur désir de raconter des histoires, les a rarement conduits à se débarrasser des pièges des films de genre. Les ouvres baroquisantes choisies ne se placeraient ni à l'intérieur d'une esthétique de la transparence (cinéma classique) ni dans les limites d'une esthétique autoréflexive, manifestement métalinguistique (cinéma moderne). Elles appartiendraient plutôt à une esthétique de la simulation, centrée sur l'artifice, où la fascination pour la matière n'implique point la simple répétition des modèles réalistes.

Le baroquisme de Visconti va depuis la configuration fugace du décor lentement composé jusqu'à l'interprétation des rôles

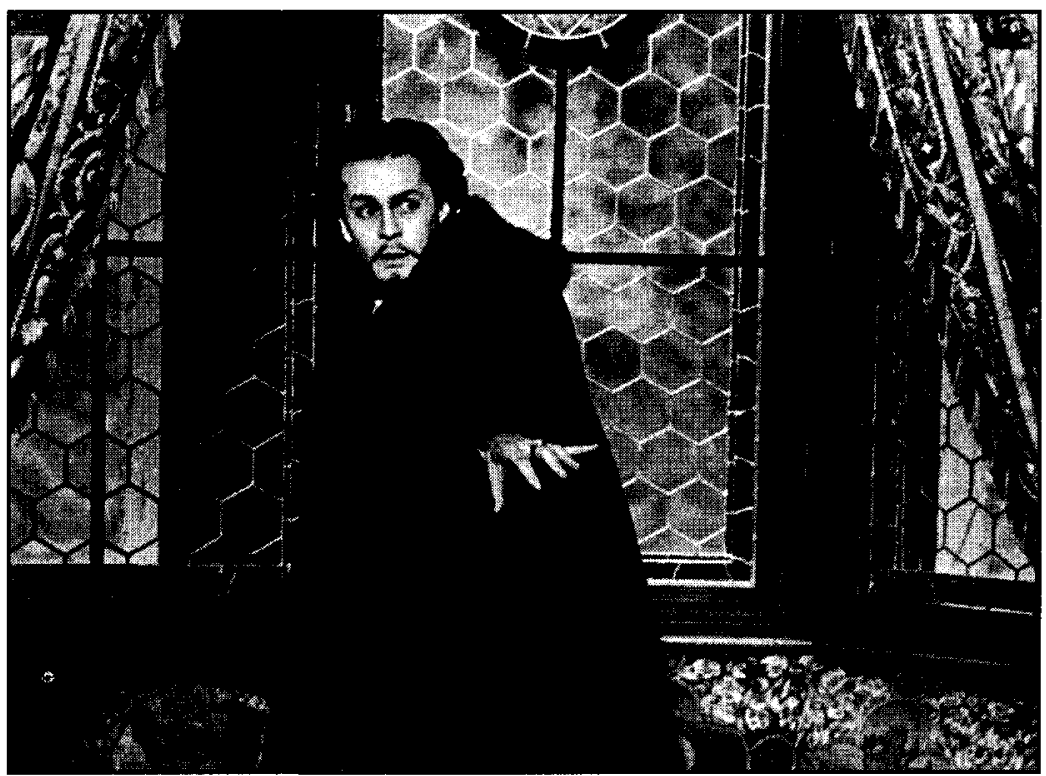

\section{Ludwig de Luchino Visconti (1972)}

Collection Cinémathèque québécoise

En deçà et au-delà du cinéma moderne. Visconti, mélancolie et néo-baroque 
théâtralisés. Ces films centrés sur les protagonistes, des acteursdivas, mettent la mort en scène dès le début. À la place de l'humanisme, déjà vu dans le manifeste de Visconti pour un cinéma anthropormophique, prend corps un cinéma des matérialités, des interprétations physiques, de la fascination pour les lieux et de l'attention portée aux objets. L'intégration entre l'espace et le temps fait des maisons et des palais une image chronotopique et matérielle. Cette image ne s'avère pas qu'une simple métaphore, figure de langage, symbole, conjugant la fascination, le charme du moment présent et l'épaisseur sociohistorique, le sensible et l'intelligible. Au contraire, le chronotope devient structurateur à la fois du tissu social et des œuvres artistiques, opérant une fusion matérielle de l'espace et du temps, en les prenant pour des catégories de connaissance. Le chronotope installe un espace de comparaison, de convergence parmi les divers phénomènes sociaux, mais il ne les rend pas pour autant homogènes, il assure leurs singularités moyennant plusieurs motifs de leurs apparitions concrètes dans chaque texte. La matérialité de ces chronotopes revient à son tour comme un désir de bâtir une pensée de la dispersion, ni duelle ni totalisante, qui renvoie à des images non abstraites, non originaires, performatives et politiques. L'image de la maison, entendue par Bachelard comme tout espace habité, possède trois sens baroques. Il s'agit d'un labyrinthe, créé surtout par le déploiement dans le temps où les personnages se retrouvent perdus, mais associés à ces sens isomorphiquement. Les espaces rongés socialement et imaginairement par le temps catastrophique se transforment en ruines, dépositaires des traces du passé, où la vie se traduit par un theatrum mundi, dont la ritualisation et l'esthétisation ne cachent pas la fragilité de l'existence des personnages confrontés au passage du temps vers la mort.

Dans cette perspective, le regard posé sur Visconti produit l'effet critique de déboulonner l'art moderne comme monument. Il ne s'agit pas, pour autant, de créer des slogans simplificateurs ( $\AA$ bas Godard! Vive Visconti!), mais d'utiliser Visconti pour réfléchir aux problématiques contemporaines qui peuvent ne pas être aussi visibles dans la production artistique. $\mathrm{Ou}$ encore, si ces problématiques ne sont que trop visibles, elles 
n'ont pas réussi à entretenir un dialogue à la hauteur de leur critique. Le dialogue entretenu par une critique qui a fait de l'avant-garde un académisme, et dont les jugements de goût se sont cristallisés, se trouve enfermé en des modèles esthétiques et ne se tourne vers la production contemporaine que pour y identifier des valeurs telles que l'originalité, la radicalité, l'utopie, la transgression. Si de telles valeurs ne se présentent pas, cette critique trouve moins de mérite à la production artistique et l'accuse de décadence, ou pire encore, elle se tait tout simplement.

Dans ce cadre, l'anachronie chez Visconti prend parfois plusieurs formes et toutes font semblant de renvoyer à la scission entre cinéma classique et cinéma moderne, ou à celle de la mimesis comme imitatio et de la mimesis comme représentation (Ishaghpour, p. 22), dont l'équivalent en littérature serait placé à distance des grands récits du roman romantico-réaliste et des œuvres de la haute modernité des premières décennies de ce siècle. Le goût pour le récit est une des anachronies qui ne s'altère pas tout au long de l'œuvre de Visconti, jusqu'à sa mort, mais dont la problématique intéresse une partie significative de la production artistique contemporaine. Celle-ci, sans oublier l'accent expérimental, est obligée de surmonter la difficulté qui consiste de nos jours à raconter une histoire. Selon Michel Buttet, dans son analyse de la séquence où Ludwig rencontre Elisabeth s'entraînant à l'équitation, l'espace référentiel d'une esthétique de la transparence, valorisant la totalité et la continuité, est absorbé par une esthétique de la représentation, marquée par la discontinuité du réel et l'autonomie d'un système symbolique. Mais, confirmant la situation d'intervalles d'au moins quelques films de Visconti,

\begin{abstract}
[...] le référent ne s'absente pas dans l'autoréflexion d'un pur langage; la forme ne nie pas l'idéologie. L'esthétique, par sa congruance à l'univers représenté, institue un autre type de "transparence". Dans cet univers où les acteurs ne vivent que de et par la représentation, le film est aussi "vitrine de l'histoire" (Buttet, p. 68).
\end{abstract}

Cela désigne un débat extrêmement important pour les années quatre-vingt, celui d'un "néo-réalisme» où les personnages habitent dans la réalité des simulacres. Le choix de Visconti

En deçà et au-delà du cinéma moderne. Visconti, mélancolie et néo-baroque 
laisse donc entrevoir une possibilité, non celle de simples répétitions de modèles prémodernistes, mais celle d'une implantation d'un dialogue subtil entre cinéma, peinture, musique et littérature, ou autrement dit, entre image, son et parole, sans jamais tenir tout à fait à l'écart un public intéressé aux spectacles et aux récits. (Néanmoins, nous sommes obligés de reconnaître la difficulté qu'a un public contemporain, marqué par la vitesse de la télévision et de la vidéo, à concentrer son attention sur ces longs plans silencieux). Quand bien même Visconti aurait été un traditionaliste, il nous intéresse encore aujourd'hui, car «[...] il pensait que n'importe qui pouvait regarder un film selon sa sensibilité et ses capacités de lecture; que le "sérieux" ne s'opposait pas au "grand public" "(Buttet, p. 171), ni la magie du faste à la somptuosité d'un grand spectacle au cinéma, ce qui l'a isolé des avant-gardes des années soixante (p. 181) marquées par des métalangages explicites (p. 184).

Le lien de son œuvre au passé s'avère exemplaire pour toute recherche d'un dialogue pluriel avec le temps qui ne procéderait pas à un collage incessant et inconsistant de quelque période historique, signe d'un pastiche dilué, qui en est venu à se constituer pour quelques-uns comme la principale caractéristique de la postmodernité en art. En ce sens, la lecture néo-baroque de quelques films de Visconti prend une distance par rapport à une vision nostalgique ou mythique de la réalité et réaffirme une historicité soit dans un sens benjaminien, pour qui le temps discontinu porte toujours en lui-même son passé ou son présent, soit dans un sens gramscien (Gramsci qui a beaucoup marqué le début de la carrière cinématographique de Visconti) où " [...] toute histoire est anachronique, le présent est histoire, et l'histoire est toujours au présent" (Ishaghpour, p. 177). Visconti ne veut pas en finir avec la tradition, mais la suivre, de la même façon qu'il la ronge (p. 25-26). En définitive, une bonne partie de l'œuvre de Visconti n'est pas faite pour des spectateurs ou pour des époques marqués par l'enthousiasme ou par l'utopie, on pourrait dire que son travail trouve plus d'écho dans les années soixante-dix et quatre-vingt que dans les années soixante, les dernières décennies étant marquées par la désillusion et le scepticisme face aux grands récits (p. 185). Finalement la cons- 
truction du temps, particulièrement de ses derniers films, sacralise le quotidien lui-même. Visconti y met en scène ses rituels, dans une "[...] somptueuse éducation de l'œil et une illustration plastique du discours" (Fieschi, p. 15). Visconti n'est pas tant un cinéaste matérialiste que celui de la matière.

Dans la mélancolie, le temps devient esthétique. Sa fugacité amplifie au lieu de réduire la fascination pour un monde d'apparences volatiles. La mélancolie émerge comme le corollaire d'une écriture amoureuse; elle se fait, plus qu'amour et beauté, désir d'amour et de beauté, la base pour une éthique esthétique aristocratique. Elle se dresse contre l'éthique protestante et bourgeoise du travail qui érige le marché comme valeur centrale, en même temps qu'elle marque sa différence avec une éthique de la dépense, qui porte encore des illusions à propos du pouvoir transgressif de la jouissance et de tout excès.

Sensibilité de l'intervalle, la mélancolie se trouve placée entre une rationalité triste et une extase momentanée dans la douleur et par-delà la douleur. J'ai commencé cet article avec l'image d'un visage et je voudrais le terminer avec celle d'un autre visage, celui de Tadzio. À la fin du film Mort à Venise (1971), un gros plan de son visage émerge un bref instant, laissant en suspens deux séquences du film: le présent de Gustav von Aschembach à Venise et ses souvenirs. Fort probablement le désir de retenir un peu plus les images de la perte, de vouloir leur donner de l'épaisseur, et de nous donner le temps de nous attarder à chaque détail de ce visage, aurait poussé Visconti à faire surgir cette image en gros plan. Ces instants, images photographiques ou portraits, tentent de briser la fluidité du récit, la présence de la mort, de retenir le temps, toujours en vain. Sur le visage de Tadzio, plus encore que le désir de beauté, persiste la quête d'images signifiantes confrontées à la fragilité du temps. Par-delà la douleur de la perte et l'horreur de savoir que tout ce qui est beau doit mourir, pouvoir tout de même encore rencontrer la beauté, quand se sentir chez soi est de nouveau possible, l'oubli se fait sans culpabilité, la mélancolie devient légèreté. Légèreté, voie de rédemption de la mélancolie, par la mélancolie'.

Universidade Federal do Rio de Janeiro 


\section{NOTE}

1 Le texte a été traduit du portugais par Mme Anamaria Skinner.

\section{OUVRAGES CITÉS}

Agamben, Giorgio. Stanze. Parole et Fantasme dans la culture occidentale. Paris: Rivages, 1994 .

Bachelard, Gaston. La Poétique de l'espace. Paris: P.U.F., 1957.

Benjamin, Walter. Origem do Drama Barroco Alemăo. Sao Paulo: Brasiliense, 1984.

Butter, Michel. "Ludwig: l'Histoire-Opéra", dans Michèle Lagny (direction), Théorème. Visconti. Classicisme et subversion. Paris: Sorbonne Nouvelle (1990), p. 7-26.

Buttet, Michel. "Le Manège viscontien", dans Michèle Lagny (direction), Théorème. Visconti. Classicisme et subversion. Paris: Sorbonne Nouvelle (1990), p. 30-40.

Daney, Serge et Jean-Pierre Oudart. «Le Nom de l'Auteur (à propos de la "place" de Mort à Venise)". Cahiers du cinéma, n“s 234-235 (1971-72).

Fieschi, Jacques. "Les rois, le matin ». Cinématographe, n" 91 (1983).

Freud, Sigmund. "Luto e Melancolia", Obras Psicologicas Completas, vol. XIV. Rio de Janeiro: Imago, 1974.

Ishaghpour, Youssef. Luchino Visconti. Le sens et l'image. Paris : La Différence, 1984.

Kristeva, Julia. Soleil Noir. Dépression et melancolie. Paris: Gallimard, 1987.

Matos, Olgária. Os Arcanos do Inteiramente Outro. A Escola de Frankfurt. A Melancolia e a Revolução. São Paulo: Brasiliense, 1989.

Rocha, Glauber. O Século do Cinema. Rio de Janeiro: Alhambra, 1985.

Rouanet, Sérgio Paulo. OÉdipo e o Anjo. Rio de Janeiro: Tempo Brasileiro, 1981.

Sanzio, Alain et Paul-Louis Thirard. Luchino Visconti. Lisboa: Dom Quixote, 1988. 